\title{
Wer Phytotherapeutika entwickelt, hat den ganzen Planeten zur Verfügung
}

Dr. Andreas Wenng ist seit Anfang des Jahres bei Swissmedic für die Komplementärmedizin verantwortlich, nachdem er mehrals zwei Jahrzehnte in der Industrie gearbeitet hat.

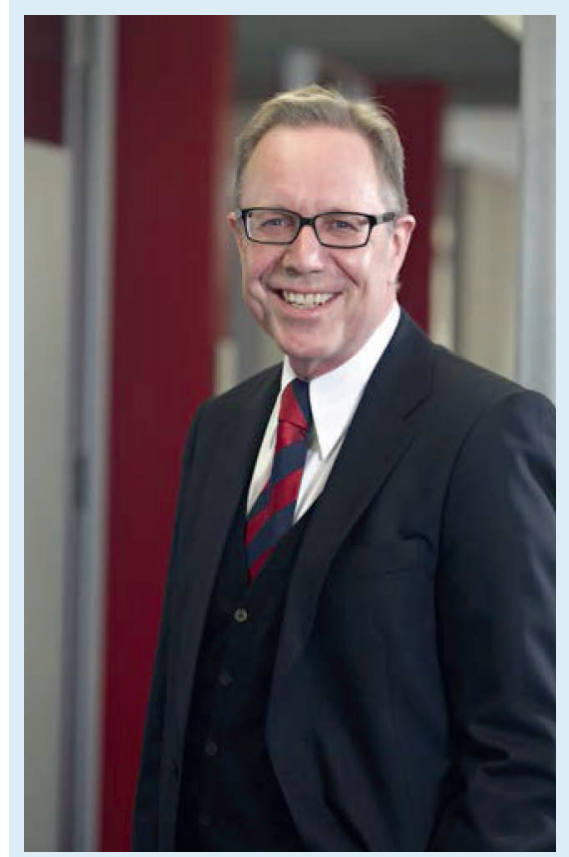

«Seitenwechsel»

Als Persönlichkeit mit langer Industrieerfahrung hat das SMPG-Mitglied Dr. Andreas Wenng Anfang des Jahres «die Seiten gewechselt». Kein Wunder, dass sein Amtsantritt bei Swissmedic mit grossem Interesse verfolgt wurde. Für die SCHWEIZERISCHE ZEITSCHRIFT FÜR GANZHEITSMEDIZIN ein Anlass, mit ihm über seine ersten Monate im Amt zu sprechen.
Ihr Stellenantritt bei Swissmedic ist mit grossen Erwartungen von Anwendern der Ganzheitsmedizin und von Industrievertretern begleitet worden. Welche müssen Sie enttäuschen?

Dr. Andreas Wenng: Die Erwartungen wurden gehegt, weil es eine Seltenheit ist, dass jemand von der Industrieauf die Behördenseite wechselt. Das war für andere genauso überraschend wie für mich übrigens auch, denn ich hätte ja in der pharmazeutischen Industrie bleiben können. Die Neugier hat mich bewogen, bei Swissmedic anzufangen, und es war ein Glücksfall, dass gerade diese Stelle offen war. Mir macht es als Biologe natürlich sehr viel Spass, auf einem so breiten Gebiet wie der Phyto- und der Komplementärmedizin bei Swissmedic tätig und ganz nahe bei der Naturwissenschaft zu sein. Weil ich mich mit botanischen Fragestellungen beschäftigen kann, ist das der Idealzustand für mich. Ich habe ein kleines Team und eine grosse Zahl von Spezialisten bei Swissmedic um mich herum. Hier im Haus gibt es einen riesigen Fundus an naturwissenschaftlichem Wissen, was mir sehr entgegenkommt. Was die Themen betrifft, ist der Blumenstrauss in meiner Abteilung reich gebunden; hierzu gehören zum Beispiel die klassischen Phytomedikamente oder die komplementärmedizinischen Produkte. Die Themengebiete der einzelnen Therapierichtungen könnten unterschiedlicher gar nicht sein das liegt mir. Ich bin seit Langem Mit- glied verschiedener phytotherapeutischer Vereinigungen, und deshalb freut es mich, dass ich für Swissmedic dieses Gebiet im Rahmen des gesetzlichen Auftrags mitgestalten kann.

Swissmedic ist ja seit einigen Monaten wegen der Einführung eines neuen Datenverarbeitungssystems im Umbruch. Swissmedic verändert sich auch deswegen laufend - und es geht in die richtige Richtung. Mir als früherem Industriemenschen geht es manchmal nicht schnell genug. Aber wenn man sich dann mal zurücklehnt, sieht man, dass die erzielten Ergebnisse sehr gut sind. Ich bin sehr zufrieden mit allem.

\section{Welche Aufgaben haben Sie nach Ihrem Amtsantritt als Erstes in Angriff genommen?}

Man wird ja immer sofort ins kalte Wasser geworfen. Wenn man den Schreibtisch in Betrieb genommen hat, muss man sich mit der Struktur des neuen Unternehmens vertraut machen. Eine Behörde ist ja anders organisiert als ein Produktionsbetrieb, wie er in den letzten Jahren mein Arbeitsort war. Ich musste mich mit den unterschiedlichen Tätigkeitsfeldern hier erst einmal auseinandersetzen. Das bedeutete, dass ich mich in die verschiedenen Verordnungen, die Gesetzestexte und die Anleitungen einlesen musste. Das Gebiet war mir nicht fremd, aber es ist voller neuer Aspekte und Fakten, sodass ich Schwerpunkte setzen musste.

\section{KARGER}

Fax +49761 4520714 
Wichtig war, die Stakeholder, die Industrievertreter, die anderen Abteilungen oder z.B. die Mitglieder der Direktion kennenzulernen.

Was selbstverständlich ganz wichtig war: Ich musste erst einmal verstehen, was meine neue Abteilung macht und auf welche Ressourcen wir innerhalb von Swissmedic zurückgreifen können. Wir bearbeiten natürlich alle Gesuche aus unserem Bereich, müssen dazu jedoch teilweise Leistungen «zukaufen» - wir brauchen klinische Reviewer und Quality Reviewer, die wir nicht selber in der Abteilung haben.

\section{Wie sind Ihre Kontakte zu den} Unternehmen, die komplementärmedizinische Erzeugnisse herstellen?

Die Industrie ist für uns natürlich ein wichtiger Stakeholder. Wir behandeln alle Unternehmen gleich und halten uns an unsere Regeln. Ich habe natürlich aufgrund meines Industriehintergrunds ein sehr offenes Verhältnis zu ihnen. Ich kenne die Sorgen und die Nöte, die sie an uns herantragen. Auch

\footnotetext{
Dr. Andreas Wenng ist seit 1. Januar 2013 Leiter der Abteilung Phyto- und Komplementärmedizin in der Abteilung Zulassung bei Swissmedic. Er hat über 20 Jahre Erfahrung in der pharmazeutischen Industrie als Herstellungsleiter Pharma gesammelt. Den grössten Teil seiner Zeit verbrachte er bei internationalen Unternehmen in der Schweiz wie Baxter, B. Braun Medical und Schott Glas. In seiner über sechsjährigen Tätigkeit als Leiter Consulting in seinem eigenen Unternehmen und bei der Firma Chemgineering widmete er sich intensiv GMP(Good Manufacturing Practice)-Fragestellungen im Produktions- und Qualitätsumfeld. In diesem Zusammenhang betreute er Phytopharma-Firmen im In- und Ausland. Dr. Andreas Wenng promovierte an der Universität Freiburg i.Ü. im Bereich Pflanzenphysiologie und startete 1989 seine Karriere bei Baxter in Düdingen nahe Bern. Er ist verheiratet und lebt - mit zwei kurzen Unterbrüchen seit 20 Jahren in der Schweiz.
}

Verbände sind wichtige Stakeholder. Bei einer ganzen Reihe dieser Organisationen habe ich mich vorgestellt und mir ein Bild davon gemacht, welche Themen den Leuten unter den Nägeln brennen. Wir fangen wieder an, Workshops mit den Verbänden $\mathrm{zu}$ machen, bei denen es z.B. um homöopathische oder phytomedizinische Therapierichtungen geht. Wir laden ein, hören uns die Probleme an, schreiben diese auf und schauen, was wir im Gesetzesrahmen für unsere Stakeholder leisten und umsetzen können.

\section{Was sind die grössten Probleme der Hersteller von komplementär- medizinischen Präparaten?}

Das sind immer die gleichen - sie drehen sich um Fristen und Kosten. Die Fristen sind ja festgelegte Zeitrahmen, bei denen wir uns im europäischen Vergleich überhaupt nicht verstecken müssen. Wir sind z.B. bei der Kommunikation von Zulassungsverfahren transparent und offen. Ich finde es wichtig, dass wir den Unternehmen Planungssicherheit geben. Schliesslich hängt deren Produktions- und Marketingplanung davon ab, wann das Zulassungsverfahren in etwa abgeschlossen sein wird. Nicht nur in dieser Beziehung verstehen wir uns als Dienstleister. Für uns bedeutet das konkret, dass wir ein verlässlicher und berechenbarer Partner für die Industrie sein möchten.

\section{Wie steht die Schweiz bei den Kosten da, die für die Zulassung fällig werden?}

Was die Zulassungskosten anbelangt, also die Gebühren, die wir von Swissmedic aus in Rechnung stellen, ist die Industrie sehr gut bedient. Viel niedriger geht es nicht. Das sind Beträge, die sich im Bereich zwischen 3000 und 6000 Franken bewegen. Vorgelagert sind die Kosten der klinischen Studien, die allerdings nur für einen
Bruchteil der komplementärmedizinischen Arzneimittel verlangt werden. Wenn man den Akteuren aus der Industrie Glauben schenken darf, liegen die in einer Grössenordnung zwischen 100000 und 150000 Franken. Ich glaube, die Industrie darf damit sehr zufrieden sein.

Die Hersteller von komplementärmedizinischen Präparaten beklagen, dass die Anforderungen und damit auch die Kosten immer stärker steigen. Stimmt das?

Wenn Sie die komplementärmedizinischen Präparate ansprechen, muss ich Ihnen nach meiner kurzen Erfahrung hier in der Behörde widersprechen. In der Schweiz haben wir eine einzigartige Situation. Die Komplementärmedizin ist in der Verfassung in Artikel 118a verankert. Damit hat sie eine Stellung, die es sonst nirgends gibt. Sie spiegelt sich auch in den verschiedenen Verordnungen wider - extra für die Komplementärmedizin ist eine Komplementär- und Phytoarzneimittel-Verordnung (KPAV) speziell zugeschnitten worden. Wir haben elektronische Verfahren zur Einreichung der Dossiers. Wir haben vereinfachte Verfahren bei der Zulassung. Da gibt es meiner Meinung nach keine Hürde. Wir sind natürlich an das Gesetz gebunden, aber das lässt der Industrie sehr viel Freiraum.

\section{Welche Bedeutung haben die Vorschriften der Europäischen Union (EU) für die Zulassung von Präparaten in der Schweiz?}

Hier gilt selbstverständlich Schweizer Recht. Wir sind aber nicht eine Art Satellit auf irgendeiner losgelösten Umlaufbahn oder eine Art Insel im Nichts. Wir haben rege Kontakte zur EU auf ganz verschiedenen Plattformen. Wir übernehmen bestimmte Dinge von der EU, wenn es Sinn hat. Bei vereinfachten Verfahren berück- 


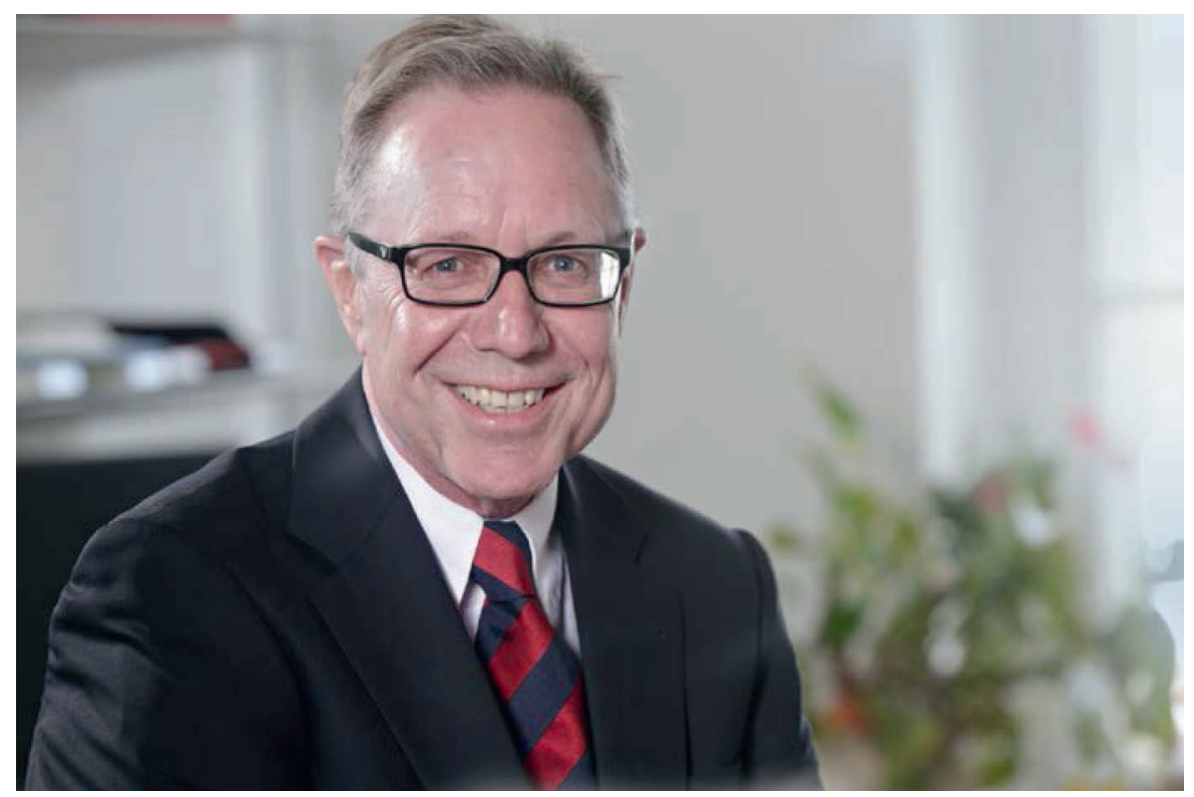

sichtigen wir, ob etwas bereits in der EU zugelassen ist; wenn ja, schauen wir, ob man diese Entscheidung in die Zulassung mit einfliessen lassen kann, um so das Verfahren noch einmal zu beschleunigen. Natürlich sind wir nicht auf die EU fixiert. Wir haben jetzt z.B. ein Konsortium mit den Behörden aus Australien, Kanada und Singapur. Wir arbeiten eng zusammen und lernen voneinander.

Wie stark hören ausländische

Behörden auf Swissmedic, was den komplementären Bereich betrifft?

Wir haben natürlich schon einen besonderen Status, wenn man jetzt an die EU denkt, weil wir eben nicht dazugehören. Wir werden trotzdem in allen wichtigen Gremien gehört, was die Komplementärmedizin anbelangt. Swissmedic ist ein sehr gefragter Partner, und ich weiss, dass wir renommiert sind und einen guten Ruf besitzen. Wir sind bei Fachtagungen vertreten und bringen uns selber mit Vorträgen ein. Ich glaube, dass man Standards sogar von uns übernehmen und in die EU einlagern könnte. Die Swissmedic hat international durchaus eine Nischenkompetenz in der Komplementärmedizin.
Nahrungsergänzungsmittel, für die es keine Zulassung braucht, machen Phytotherapeutika Konkurrenz.

Unternehmen beklagen, dass ihr Geschäft schwieriger wird. Wie sehen Sie das?

Was draussen am Markt passiert, ist das Ergebnis von zwei grossen Umwälzungen, die schon relativ alt sind. Das ist einmal die Globalisierung mit dem Internet und das ist ausserdem eine boomende Nutraceutical-Industrie, die mit ihren Produkten auf den Markt drängt. Diese negative Entwicklung zu bekämpfen und zu regulieren ist nicht Aufgabe meiner Abteilung; hierfür gibt es andere Instanzen. Wir warnen aber als Swissmedic explizit vor dem Kauf von Präparaten durch das Internet. Im Grossen und Ganzen werden wir Zeuge eines Trends, den man auch nicht mehr umkehren kann. Die Aufgabe, das Beste aus dieser Situation zu machen, hat die Industrie, die sich gut positionieren und gegen die Konkurrenz ohne Zulassung auf dem Markt abgrenzen muss. Wenn sie von Swissmedic zugelassene Produkte auf dem Markt hat, besitzen diese ein hohes Mass an Sicherheit und Qualität. Bei den Nahrungsergänzungsmitteln, die sich Leute von irgendwoher im Internet bestellen, sind die Qualitätsstandards oft zweifelhaft - und die Produkte somit unter Umständen gesundheitsgefährdend. ein. Aber unsere Kleinheit hat Vorteil, dass wir uns relativ schnell austauschen, dass wir sehr flexibel reagieren und dass wir auch rasch mit externen Beratern zusammenarbeiten können, wenn wir Know-how von aussen brauchen. Als kleine Gruppe können wir natürlich nicht alles selber leisten. Mit den externen Beratern sind wir in der Lage, Belastungsspitzen aufzufangen. Wir haben ein System, das uns hilft, für eine bestimmte Aufgabe die entsprechenden Berater auszuwählen, die uns dann zuarbeiten und helfen.

\section{Wie sieht es mit der Entwicklung neuer Medikamente in der Komple- mentärmedizin aus? Hersteller beklagen, dass durch sehr hohe Anforderungen an Sicherheit und Wirksamkeit die Forschung und Entwicklung vieler mittelständischer Unternehmen ausgebremst werde.}

Wir sprechen in der Schweiz von etwa 40 mittelständischen Unternehmen, die im letzten Jahr nur zwei wirklich innovative Produkte bei uns zur Zulassung eingereicht haben, bei denen 
Indikation und Wirkstoffe neu waren. Das ist natürlich wenig. Aber Sie dürfen die Hersteller von Phytotherapeutika nicht mit denen von synthetischen Heilmitteln vergleichen, die ihre Wirkstoffe selber erfinden müssen. Wer auf pflanzliche Wirkstoffe setzt, hat im Prinzip den Planeten zur Verfügung, aus dem er schöpfen kann, weil alles, was wächst, in irgendeiner Art und Weise verwertet werden kann. Die Chancen sind riesig, etwas Neues zu finden. In der Tat können die Anforderungen der Zulassung eine Hürde sein, die allerdings nicht Swissmedic zu verantworten hat, sondern das Parlament. In der zweiten Revision des Heilmittelgesetzes, die jetzt diskutiert wird, soll es kleine Vereinfachungen geben. Ich glaube, dass es im Grossen und Ganzen für die Mittelständler machbar ist, neue Produkte auf den Markt zu bringen. Man muss die Innovationen aber auch in Beziehung zum Gesamtangebot sehen. Insgesamt sind in der Schweiz über 12000 Präparate zugelassen. Damit ist ein ganzes Bündel an Präparaten auf dem Markt. Und man darf nicht vergessen, dass sich die Komplementärmedizin durch die Individualtherapie auszeichnet, die nicht unbedingt neue Wirkstoffe braucht.

\section{Was ist für Sie als gelernter Biologe die interessanteste Heilpflanze?}

Hanf. Weil Sie aus Hanf so viel machen können. Jenseits der Wirkung als Droge hat diese Pflanze ein faszinierendes Einsatzspektrum. Aus der Hanffaser können Sie Textilien machen, Sie können sie als Baustoff ver- wenden oder als Futtermittelzusatz verfüttern oder als Dünger einsetzen. Sie haben eine Vielfalt an Möglichkeiten, was Sie mit dieser Pflanze machen können. Sie ist leicht $\mathrm{zu}$ ziehen und wächst auch bei uns. Mit Hanf können Sie im Prinzip Ihr Leben verbringen und sich noch kleiden lassen. Die Anwendungen in der Medizin sind natürlich sehr interessant. Wenn Sie an die Indikationen denken, die in der Literatur diskutiert werden, z.B. als Schmerzmittel bei der Behandlung von Multipler Sklerose, dann finde ich das einfach fantastisch. Es ist schade, dass Hanfbeispielsweise von den Medien immer nur als Droge verteufelt wird. Wichtige andere Themen zu dieser Pflanze werden so leider aus dem Fokus gedrängt.

\section{Wie sehen Sie die Zukunft der Komplementärmedizin und ihrer Heilmittel in der Schweiz?}

Wir versuchen, Trends zu beobachten, die auch für unsere eigene Strategie wichtig sind. Wir verfügen aber über kein verlässliches Zahlenmaterial, das uns hilft, die heutige Marktsituation richtig $\mathrm{zu}$ beurteilen, und auch über keine halbwegs gesicherten Annahmen, wie sich der Markt in Zukunft entwickelt. Meine Einschätzungen beruhen deshalb etwas auf dem Bauchgefühl. Ich glaube, was in der Schweiz noch unterentwickelt und noch weiter im Kommen ist, sind Wirkstoffe und Präparate aus der Traditionellen Chinesischen Medizin. Ich erwarte, dass wir hier in Zukunft Neues auf dem Markt sehen werden.
Auch nach einem halben Jahr bereuen Sie nicht, zu Swissmedic gegangen zu sein. Sind Sie immer noch motiviert?

Auch wenn das Wort «Stolz» verpönt ist, muss ich doch sagen, dass ich stolz bin, für diese Behörde $z u$ arbeiten. Toll ist die Dynamik, die von der Direktion vorgegeben ist. Was die Managementtools betrifft, sind wir absolut fortschrittlich. Da kann sich manches Industrieunternehmen eine Scheibe abschneiden. Die Menschenführung hier ist modern und jeder wird individuell betrachtet. Das alte IKS(Internes Kontrollsystem)-Denken der Vorläuferorganisation soll verschwinden und ist kaum noch vorhanden. Silodenken ist bei uns nicht mehr gefragt, sondern wir wollen interagieren und Netzwerke bilden. Wir möchten uns auf den verschiedenen Ebenen austauschen.

Wir wollen uns natürlich auch international gut darstellen. Wir möchten mit den Behörden z.B. in Brasilien, Kanada oder Japan Gespräche auf Augenhöhe führen können. Unser Ziel ist es, uns als hochwertiger Partner in und aus der kleinen Schweiz weiterzuentwickeln, und ich bin überzeugt, dass wir das schaffen. Wir sind auf dem besten Weg. Und wenn Sie meine Motivation ansprechen: Ich war immer schon motiviert, und da wird es kaum Abstriche geben.

Interview: Oliver Klaffke 